\title{
Hamilton's Turns for the Lorentz Group
}

\author{
R.Simon* \\ The Institute of Mathematical Sciences, C. I. T. Campus, Chennai 600 113, India \\ S. Chaturvedi ${ }^{\dagger}$ \\ School of Physics, University of Hyderabad, Hyderabad 500 046, India \\ V. Srinivasan $\ddagger$ \\ Department of Theoretical Physics, University of Madras, Guindy Campus, Chennai 600025 \\ N. Mukunda ${ }^{\S}$ \\ Centre for High Energy Physics, Indian Institute of Science, Bangalore 560 012, India
}

(November 16, 2017)

\begin{abstract}
Hamilton in the course of his studies on quaternions came up with an elegant geometric picture for the group $S U(2)$. In this picture the group elements are represented by "turns", which are equivalence classes of directed great circle arcs on the unit sphere $S^{2}$, in such a manner that the rule for composition of group elements takes the form of the familiar parallelogram law for the Euclidean translation group. It is only recently that this construction has been generalized to the simplest noncompact group $S U(1,1)=S p(2, R)=S L(2, R)$, the double cover of $S O(2,1)$. The present work develops a theory of turns for $S L(2, C)$, the double and universal cover of $S O(3,1)$ and $S O(3, C)$, rendering a geometric representation in the spirit of Hamilton available for all low dimensional semisimple Lie groups of interest in physics. The geometric construction is illustrated through application to polar decomposition, and to the composition of Lorentz boosts and the resulting Wigner or Thomas rotation.
\end{abstract}

PACS: 02.20.-a

\section{INTRODUCTION}

The group $S U(2)$ plays an important role in various branches of physics. One is generally familiar with two ways of parametrizing the elements of $S U(2)$ - the Euler angle parametrization and the Cayley-Klein parametrization. In neither of these parametrizations is the expression for the group composition law particularly illuminating: there is no simple way of remembering or visualizing the composition law. Hamilton [1], in the course of his studies on quaternions, developed an interesting geometric picture for representing the elements of $S U(2)$ wherein the group composition law acquires the structure of the familiar head to tail parallelogram rule of vector addition. This work has come to be known as Hamilton's theory of turns, and an excellent review can be found in the monograph of Biedenharn and Louck [2]. Interestingly, this elegant geometric picture does not seem to be as well known as it deserves, and it is only recently that Hamilton's construction was generalized to the simplest noncompact semisimple Lie group $S U(1,1)[3,4]$.

The ideas underlying Hamilton's construction can be best and most easily understood through analogy with the much simpler case of the Abelian group of translations in a three dimensional Euclidean space. Each element of this group can be thought of as a unique point in a three dimensional space or, equivalently, as a vector emanating from the origin - the point representing the identity element. The composition law for this group then corresponds to simply adding vectors representing the group elements using the parallelogram law.

Examined in detail, the parallelogram law for the translation group involves giving up the picture in the last paragraph wherein each element of the group was represented by a vector with tail pegged to the origin, and going over to a picture based on free vectors - vectors with their tails unpegged. Let $(\boldsymbol{x}, \boldsymbol{y})$ represent the free vector with head at $\boldsymbol{y}$ and tail at $\boldsymbol{x}$. The group element corresponding to translation by amount $\boldsymbol{a}$ is represented not by a

*email: simon@imsc.ernet.in

${ }^{\dagger}$ e-mail: scsp@uohyd.ernet.in

${ }^{\ddagger}$ e-mail: vsspster@gmail.com

§email: nmukunda@cts.iisc.ernet.in 
single free vector, but by the equivalence class of all free vectors $(\boldsymbol{x}, \boldsymbol{y})$ obeying the only condition $\boldsymbol{y}-\boldsymbol{x}=\boldsymbol{a}$. The parallelogram law for composing two group elements $(\boldsymbol{x}, \boldsymbol{y})$ and $(\boldsymbol{u}, \boldsymbol{v})$ in this picture simply amounts to choosing representative free vectors, one from either equivalence class, in such a manner that the head of the first free vector coincides with the tail of the second. We may use, for instance, the equivalence $(\boldsymbol{u}, \boldsymbol{v})=(\boldsymbol{y}, \boldsymbol{y}+\boldsymbol{v}-\boldsymbol{u})$. Then the product of the two group elements is the free vector from the tail of the first to the head of the second vector:

$$
(\boldsymbol{u}, \boldsymbol{v})(\boldsymbol{x}, \boldsymbol{y})=(\boldsymbol{y}, \boldsymbol{y}+\boldsymbol{v}-\boldsymbol{u})(\boldsymbol{x}, \boldsymbol{y})=(\boldsymbol{x}, \boldsymbol{y}+\boldsymbol{v}-\boldsymbol{u}) .
$$

Note that the equivalence class of free vectors corresponding to a group element is obtained from the unique vector pegged to the origin by the left action of the translation group. Thus, the above construction can be carried over to any group $G$ through left action of the group on the group manifold itself. More precisely, to each group element $g$ one can associate an equivalence class of pairs $\left(g_{0}, g g_{0}\right)$, with the tail $g_{0}$ running over the entire group manifold. It can easily be verified that whatever was said about the translation group goes through here as well. In particular, composing two group elements $g, g^{\prime}$ represented by the equivalence classes corresponding respectively to $\left(g_{0}, g g_{0}\right)$ and $\left(g_{0}^{\prime}, g^{\prime} g_{0}^{\prime}\right)$ requires us to choose the representative pair from each equivalence class in such a manner that the head $g g_{0}$ of the first one coincides with the tail of the second pair, $\left(g_{0}^{\prime}, g^{\prime} g_{0}^{\prime}\right) \sim\left(g g_{0}, g^{\prime} g g_{0}\right)$, so that the resulting equivalence class $\left(g_{0}, g^{\prime} g g_{0}\right)$ corresponds to the group element $g^{\prime} g$, thus endowing the composition law of the arbitrary group $G$ with the structure of the parallelogram rule.

This naive generalization of the parallelogram law applies uniformly to all groups, and does not take advantage of the specific features of a given group. It requires us to ascribe to each element of the group an equivalence class of pairs of points on the group manifold, and this equivalence class is exactly as large as the group manifold itself. Thus, in the case of $S U(2)$ for which the group manifold is $S^{3}$ one would be associating with each group element an equivalence class of pairs of points on $S^{3}$ (the tail point of a pair can be considered to be arbitrary, and the head point is then fixed by the tail and the group element under consideration). The importance of Hamilton's work lies in his recognizing that formulation of the $S U(2)$ composition law as a parallelogram rule can be accomplished, with greater economy, using equivalence classes of pairs of points on $S^{2}$ rather than on $S^{3}$.

The relationship between $S U(2)$ and $S^{2}$ is that the latter is an adjoint orbit of the former. That the parallelogram law for composition of $S U(2)$ group elements can be constructed on the (smaller) adjoint orbit without having to resort to the full group manifold rests ultimately on the fact that $S U(2)$ is strongly nonabelian (its centre is discrete). In comparison, since the translation group of the $n$-dimensional Euclidean space is abelian, parallelogram law for it cannot be constructed in any space smaller than the group manifold $\mathcal{R}^{n}$.

Just as $S U(2)$, the pseudo-unitary semisimple group $S U(1,1)$, which is isomorphic to the real symplectic group $S p(2, R)$ of linear canonical transformations, plays an important role in several areas of physics like squeezed light, Bogoliubov transformations, Gaussian or first order optics, transmission lines, and reflection and transmission of classical and Schrödinger waves at lossless boundaries and through barriers. Further, $S U(1,1)$ is the double cover of the (pseudo-orthogonal) Lorentz group $S O(2,1)$ of the $(2+1)$-dimensional space-time, a relationship similar to the one between $S U(2)$ and $S O(3)$.

Another low dimensional group whose importance for physics cannot be over-emphasised is the semisimple group $S L(2, C)$. This group is the double (and universal) cover of the Lorentz group $S O(3,1)$. Moreover, the complex orthogonal group $S O(3, C)$ is isomorphic to $S O(3,1)$ : under a Lorentz transformation the space-time coordinates transform as an $S O(3,1)$ vector, but the three components of $\boldsymbol{E} \pm i \boldsymbol{H}$, where $\boldsymbol{E}$ and $\boldsymbol{H}$ are the electric and magnetic field vectors, transform as mutually conjugate $S O(3, C)$ vectors.

As noted above, Hamilton's theory of turns has already been generalized to $S U(1,1)$. The purpose of the present paper is to develop a theory of turns for $S L(2, C)$ so that a geometric representation in the spirit of Hamilton will be available for all the low dimensional semisimple Lie groups of interest in physics.

The contents of this paper are organized as follows. In Section 2 we recount briefly Hamilton's theory of turns for $S U(2)$, and also its generalization to $S U(1,1)$. This summary should prove useful in view of the fact that the theory of turns that we develop for $S L(2, C)$ runs almost parallel to that in the above cases. The adjoint orbits in the Lie algebra of $S L(2, C)$ are considered in Section 3, and the relationships between the groups $S O(3,1), S O(3, C)$ and $S L(2, C)$ are indicated. In Section 4 we construct turns for $S L(2, C)$ in the adjoint orbit of complex "unit" vectors $\Sigma=\left\{\widehat{\boldsymbol{z}}=\left(z_{1}, z_{2}, z_{3}\right), z_{1}^{2}+z_{2}^{2}+z_{3}^{2}=1\right\}$, and demonstrate the parallelogram law for composition of turns. As an exercise in the use of turns, in Section 5 we describe the polar decomposition in the language of turns. Composition of Lorentz boosts and the resulting Wigner (Thomas) rotation are studied in Section 6 using turns. We conclude in Section 7 with some final remarks. 


\section{TURNS FOR $S U(2)$ AND $S U(1,1)$}

In the defining $2 \times 2$ representation, elements of the group $S U(2)$ are described in terms of the Pauli matrices $\boldsymbol{\sigma}=\left(\sigma_{1}, \sigma_{2}, \sigma_{3}\right)$ as

$$
g\left(a_{0}, \boldsymbol{a}\right)=a_{0}-i \boldsymbol{a} \cdot \boldsymbol{\sigma},
$$

where $a_{0}$ is a real scalar and $\boldsymbol{a}$ is a real three vector satisfying the constraint

$$
a_{0}^{2}+\boldsymbol{a} \cdot \boldsymbol{a}=1 .
$$

Thus the group manifold of $S U(2)$ is the unit sphere $S^{3}$. The centre of $S U(2)$ is $Z_{2}$, the subgroup consisting of the two elements \pm 1 .

The Lie algebra considered as a linear space coincides with $\mathcal{R}^{3}$, and it consists of all traceless hermitian matrices $\boldsymbol{x} \cdot \boldsymbol{\sigma}, \boldsymbol{x} \in \mathcal{R}^{3}$. The group $S U(2)$, modulo its center $Z_{2}$, can be realized by its adjoint action on its Lie algebra:

$$
\begin{aligned}
g: \quad \boldsymbol{x} \cdot \boldsymbol{\sigma} \rightarrow g \boldsymbol{x} \cdot \boldsymbol{\sigma} g^{-1}=\boldsymbol{x}^{\prime} \cdot \boldsymbol{\sigma} \\
\quad \boldsymbol{x}^{\prime}=R(g) \boldsymbol{x}, \quad R(g) \in S O(3)=S U(2) / Z_{2} .
\end{aligned}
$$

Thus $\boldsymbol{x} \cdot \boldsymbol{x}=-\operatorname{det} \boldsymbol{x} \cdot \boldsymbol{\sigma}$ is invariant, and hence the adjoint orbits of $S U(2)$ are spheres centred at the origin. Hamilton's turns can be constructed on any of these orbits, and we choose the unit sphere $S^{2}$.

The construction goes as follows. Given $g\left(a_{0}, \boldsymbol{a}\right) \in S U(2)$, choose a pair of unit vectors $\widehat{\boldsymbol{x}}, \widehat{\boldsymbol{y}} \in S^{2}$ such that $a_{0}=\widehat{\boldsymbol{x}} \cdot \widehat{\boldsymbol{y}}, \boldsymbol{a}=\widehat{\boldsymbol{x}} \wedge \widehat{\boldsymbol{y}}$. This is always possible and, indeed, there exists not just one choice but an equivalence class of choices. When $\boldsymbol{a} \neq 0$, the unit vectors $\widehat{\boldsymbol{x}}, \widehat{\boldsymbol{y}}$ are necessarily orthogonal to $\boldsymbol{a}$, and there exists a one parameter worth of freedom in the choice of $\widehat{\boldsymbol{x}}, \widehat{\boldsymbol{y}}$ : for instance, $\widehat{\boldsymbol{x}}$ can be chosen to be an arbitrary point on the great circle of $S^{2}$ perpendicular to $\boldsymbol{a}$, and then $\widehat{\boldsymbol{y}}$ is uniquely determined by $\widehat{\boldsymbol{x}} \wedge \widehat{\boldsymbol{y}}=\boldsymbol{a}$ and $\widehat{\boldsymbol{x}} \cdot \widehat{\boldsymbol{y}}=a_{0}$. On the other hand, when $\boldsymbol{a}=0$, i.e. when $g\left(a_{0}, \boldsymbol{a}\right)= \pm 1$, the vectors $\widehat{\boldsymbol{x}}, \widehat{\boldsymbol{y}}$ are necessarily parallel or antiparallel, and there is a two parameter worth of freedom in choosing the pair ( $\widehat{\boldsymbol{x}}$ may be chosen to be an arbitrary point, and then $\widehat{\boldsymbol{y}}$ is fixed: $(\widehat{\boldsymbol{x}}, \widehat{\boldsymbol{y}})=(\widehat{\boldsymbol{x}}, \pm \widehat{\boldsymbol{x}}))$.

We may use $\widehat{\boldsymbol{x}}, \widehat{\boldsymbol{y}}$ to label the elements of $S U(2)$ and write

$$
g(\widehat{\boldsymbol{x}}, \widehat{\boldsymbol{y}})=\widehat{\boldsymbol{x}} \cdot \widehat{\boldsymbol{y}}-i \widehat{\boldsymbol{x}} \wedge \widehat{\boldsymbol{y}} \cdot \boldsymbol{\sigma} .
$$

This equation describes a one-to-one correspondence between elements of $S U(2)$ and equivalence classes of pairs of points in $S^{2}$.

Now, a pair of points in $S^{2}$ is the same thing as a directed great circle arc with tail at the first member of the pair and head at the second. Thus emerges the one-to-one correspondence between elements of $S U(2)$ and equivalence classes of directed great circle (geodesic) arcs. These equivalence classes are the turns of Hamilton. Arcs of an equivalence class belong to the same great circle, have the same sense and same arc length $\leq \pi$, so that the members of an equivalence class are obtained by rigidly sliding one arc of the class along its own geodesic. The element $-1 \in S U(2)$ corresponds to the equivalence class of great semicircles or antipodal points, and the identity element to the equivalence class of null arcs or singleton points.

Since $g\left(a_{0}, \boldsymbol{a}\right)^{-1}=g\left(a_{0},-\boldsymbol{a}\right)$, we have $g(\widehat{\boldsymbol{x}}, \widehat{\boldsymbol{y}})^{-1}=g(\widehat{\boldsymbol{y}}, \widehat{\boldsymbol{x}})$. That is, the $S U(2)$ inverse of a turn corresponds simply to reversal of the sense of the turn. The group composition law rests on the identity

$$
g(\widehat{\boldsymbol{y}}, \widehat{\boldsymbol{z}}) g(\widehat{\boldsymbol{x}}, \widehat{\boldsymbol{y}})=g(\widehat{\boldsymbol{x}}, \widehat{\boldsymbol{z}}),
$$

which follows from the definition (2.4). This is the parallelogram law for the product of two $S U(2)$ elements when the tail of the left factor coincides with "and cancels" the head of the right factor at $\widehat{\boldsymbol{y}}$. The product then corresponds to the directed great circle arc from the free tail $\widehat{\boldsymbol{x}}$ to the free head $\widehat{\boldsymbol{z}}$, as shown in Fig.1.

That it is always possible, given two arbitrary elements of $S U(2)$, to choose directed arcs from the respective turns so that the tail of the left element coincides with the head of the right element is guaranteed by the fact that great circles on $S^{2}$ intersect (or coincide). Thus, the parallelogram law (2.5) faithfully reproduces $S U(2)$ multiplication (including its noncommutativity!). 


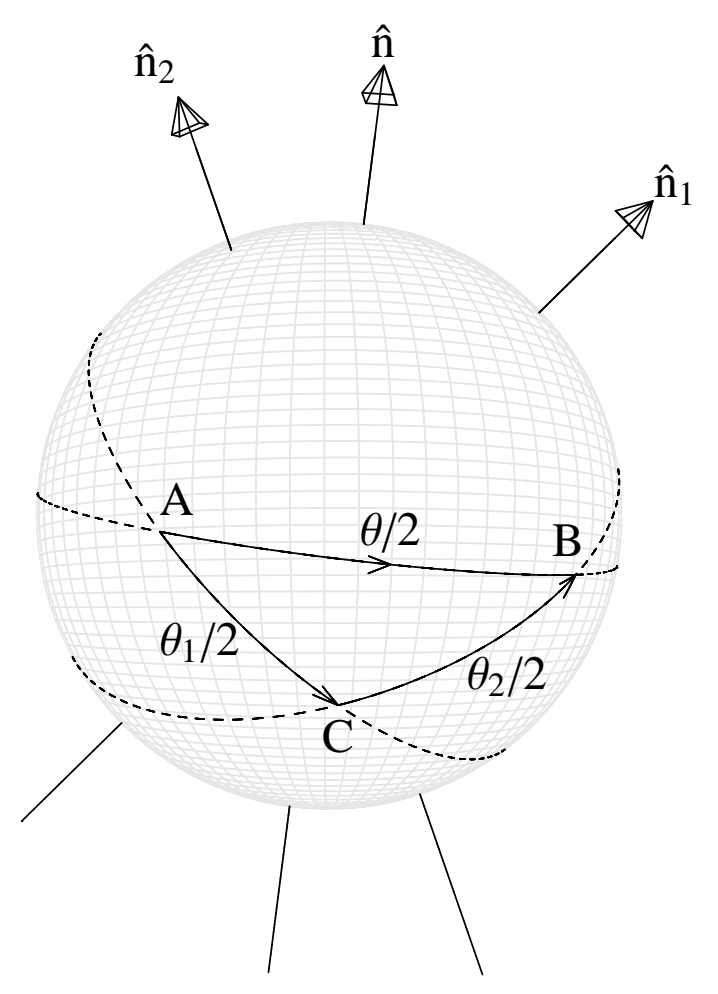

FIG. 1. Showing the parallelogram law of group composition for $S U(2)$. The turns representing $S\left(\theta_{1}, \widehat{\boldsymbol{n}}_{1}\right)$ and $S\left(\theta_{2}, \widehat{\boldsymbol{n}}_{2}\right)$ live in the equators orthogonal to $\widehat{\boldsymbol{n}}_{1}$ and $\widehat{\boldsymbol{n}}_{2}$ respectively. The head of $S\left(\theta_{1}, \widehat{\boldsymbol{n}}_{1}\right)$ and the tail of $S\left(\theta_{2}, \widehat{\boldsymbol{n}}_{2}\right)$ meet at C. The directed geodesic arc AB from the free tail of $S\left(\theta_{1}, \widehat{\boldsymbol{n}}_{1}\right)$ to the free head of $S\left(\theta_{2}, \widehat{\boldsymbol{n}}_{2}\right)$ represents the turn corresponding to the product $S(\theta, \widehat{\boldsymbol{n}})=S\left(\theta_{2}, \widehat{\boldsymbol{n}}_{2}\right) S\left(\theta_{1}, \widehat{\boldsymbol{n}}_{1}\right)$, with $\widehat{\boldsymbol{n}}$ orthogonal to the geodesic $\mathrm{AB}$ and $\theta=$ twice the arclength of $\mathrm{AB}$.

Given $a_{0}-i \boldsymbol{a} \cdot \boldsymbol{\sigma} \in S U(2)$, the condition $a_{0}{ }^{2}+\boldsymbol{a} \cdot \boldsymbol{a}=1$ implies that we can find a unit vector $\widehat{\boldsymbol{n}}$ and an angle $-2 \pi<\theta \leq 2 \pi$ such that $a_{0}=\cos \theta / 2$ and $\boldsymbol{a}=\widehat{\boldsymbol{n}} \sin \theta / 2$. Thus, we may also parametrize the elements of $S U(2)$ as

$$
S(\theta, \widehat{\boldsymbol{n}})=\cos \theta / 2-i \widehat{\boldsymbol{n}} \cdot \boldsymbol{\sigma} \sin \theta / 2,
$$

rendering the fact that $S^{3}$, the group manifold of $S U(2)$, is a $U(1)$ bundle over $S^{2}$. In the adjoint representation $S(\theta, \widehat{\boldsymbol{n}})$ acts as rotation through angle $\theta$ about the direction $\widehat{\boldsymbol{n}}$. While the unit vectors $(\widehat{\boldsymbol{x}}, \widehat{\boldsymbol{y}})$ in $(2.4)$ live in the "equator" orthogonal to $\widehat{\boldsymbol{n}}$, the angle between them is $\theta / 2$ and not $\theta$ (see Fig. 1).

This concludes our account of Hamilton's theory of turns for $S U(2)$. We have gone over it in some detail, for the theory of turns that we shall develop for the Lorentz group will follow the $S U(2)$ case quite closely.

The theory of turns has turned out to be a powerful tool in connexion with several synthesis problems in polarization optics [5]. A particularly striking result in this context is the following: every intensity preserving [i.e., SU(2)] transformation in polarization optics can be synthesised with just two quarter wave plates and one half wave plate [5-8]. Moreover, an intimate connection between $S U(2)$ turns and the Berry-Pancharatnam [9] geometric phase has been established [10].

Hamilton's theory of turns has been generalized to the noncompact group $S U(1,1)$ only recently [3,4]. This group consists of two dimensional complex pseudounitary matrices of unit determinant:

$$
g=\left(\begin{array}{cc}
\alpha & \beta \\
\beta^{*} & \alpha^{*}
\end{array}\right), \quad|\alpha|^{2}-|\beta|^{2}=1 .
$$

It order to maintain a close similarity with the group $S U(2)$, it proves convenient to define

$$
\begin{aligned}
\boldsymbol{\rho} & =\left(\rho_{1}, \rho_{2}, \rho_{3}\right), \\
\rho_{1}=\sigma_{3}, \quad \rho_{2} & =i \sigma_{1}, \quad \rho_{3}=i \sigma_{2},
\end{aligned}
$$

and to introduce the $(2+1)$-Minkowskian analogues (with indices running over 1,2,3 rather than over $0,1,2$ ) of the scalar and the cross products of vectors as follows 


$$
\boldsymbol{x} \cdot \boldsymbol{y} \equiv \eta_{a b} x^{a} y^{b}, \quad \boldsymbol{x} \wedge \boldsymbol{y} \equiv \epsilon_{b c}^{a} x^{b} y^{c}
$$

where $\eta_{a b}=\operatorname{diag}(-1,+1,+1)$ is the metric, and $\epsilon_{a b c}$ is the Levi-Civita symbol with $\epsilon_{123}=1$. With the help of this notation $S U(1,1)$ matrices, in analogy with the $S U(2)$ case, can be expressed as

$$
g\left(b_{0}, \boldsymbol{b}\right)=b_{0}-i \boldsymbol{b} \cdot \boldsymbol{\rho}
$$

where the scalar $b_{0}$ and the vector $\boldsymbol{b}$ are real and are constrained by

$$
b_{0}{ }^{2}-\boldsymbol{b} \cdot \boldsymbol{b}=b_{0}{ }^{2}+b_{1}^{2}-b_{2}^{2}-b_{3}^{2}=1 .
$$

The group manifold of $S U(1,1)$ is the hyperboloid described by (2.10). Topologically it has the same structure as $\mathcal{R}^{2} \times S^{1}$, as can be seen by solving for $b_{0}$ and $b_{1}$ in $(2.10)$ in terms of $\left(b_{2}, b_{3}\right) \in \mathcal{R}^{2}$ and an angle variable $\theta$ :

$$
\begin{aligned}
& b_{0}=\sqrt{1+b_{2}^{2}+b_{3}^{2}} \cos \theta, \\
& b_{1}=\sqrt{1+b_{2}^{2}+b_{3}^{2}} \sin \theta .
\end{aligned}
$$

Thus, the group manifold of $S U(1,1)$ is noncompact. It is multiply connected, while that of the compact group $S U(2)$ is simply connected. However, the centre of $S U(1,1)$ is $Z_{2}$, the subgroup consisting of the two elements \pm 1 , just as with $S U(2)$.

The Lie algebra of $S U(1,1)$ consists of the matrices $\boldsymbol{x} \cdot \boldsymbol{\rho}, \boldsymbol{x} \in \mathcal{R}^{3}$. Adjoint action of $S U(1,1)$ on its Lie algebra gives a realization of $S U(1,1)$ modulo its center $Z_{2}$ :

$$
\begin{aligned}
g: \quad \boldsymbol{x} \cdot \boldsymbol{\rho} \rightarrow g \boldsymbol{x} \cdot \boldsymbol{\rho} g^{-1} & =\boldsymbol{x}^{\prime} \cdot \boldsymbol{\rho}, \\
\boldsymbol{x}^{\prime} & \equiv \Lambda(g) \boldsymbol{x}, \\
\Lambda(g) \in S O(2,1) & =S U(1,1) / Z_{2} .
\end{aligned}
$$

We made use of the fact that det $\boldsymbol{x} \cdot \boldsymbol{\rho}=\boldsymbol{x} \cdot \boldsymbol{x}=x_{2}^{2}+x_{3}^{2}-x_{1}^{2}$ is invariant under conjugation

Whereas all the adjoint orbits of $S U(2)$ were of the same type, namely concentric spheres, in the $S U(1,1)$ case there are different types of orbits since, owing to the indefinite nature of the Minkowskian scalar product, the invariant $\boldsymbol{x} \cdot \boldsymbol{x}=\operatorname{det} \boldsymbol{x} \cdot \boldsymbol{\rho}$ can be positive, negative, or zero (adjoint orbits of the orthogonal and pseudo-orthogonal groups $S O(m, n), m+n \leq 5$, have been analysed and classified in Ref. [11]). The single sheeted unit hyperboloid consisting of spacelike unit vectors $\boldsymbol{x}$ with $x_{2}^{2}+x_{3}^{2}-x_{1}^{2}=1$ proves convenient [3,4] in generalizing Hamilton's theory of turns to $S U(1,1)$. Indeed, the construction proceeds in close analogy with the $S U(2)$ case, and the details can be found in Refs. $[3,4]$.

\section{III. $S L(2, C)$ AND ITS CONNECTION WITH $S O(3, C)$ AND $S O(3,1)$}

The group $S L(2, C)$ in its defining representation consists of complex valued $2 \times 2$ matrices of unit determinant:

$$
S=\left(\begin{array}{ll}
a & b \\
c & d
\end{array}\right), \quad a d-b c=1 .
$$

It is a noncompact six parameter real Lie group. The centre of $S L(2, C)$ is $Z_{2}=\{ \pm 1\}$, and the group manifold is simply connected.

The Lie algebra consists of all traceless complex matrices $\boldsymbol{z} \cdot \boldsymbol{\sigma}, \boldsymbol{z} \in \mathcal{C}^{3}$. In other words the one parameter subgroups are of the form

$$
\begin{aligned}
g_{\boldsymbol{z}}(t)=\exp (-i t \boldsymbol{z} \cdot \boldsymbol{\sigma}) & =\exp [-i t(\boldsymbol{x} \cdot \boldsymbol{\sigma}+i \boldsymbol{y} \cdot \boldsymbol{\sigma})], \\
\boldsymbol{x}, \boldsymbol{y} & \in \mathcal{R}^{3}, \quad \boldsymbol{z}=\boldsymbol{x}+i \boldsymbol{y} \in \mathcal{C}^{3} .
\end{aligned}
$$

The connection between $S L(2, C)$ and $S O(3, C)$ is exposed by the adjoint action of the former on its Lie algebra:

$$
\begin{aligned}
S: \quad \boldsymbol{z} \cdot \boldsymbol{\sigma} \rightarrow S \boldsymbol{z} \cdot \boldsymbol{\sigma} S^{-1}=\boldsymbol{z}^{\prime} \cdot \boldsymbol{\sigma}, \\
\quad \boldsymbol{z}^{\prime}=R^{c}(S) \boldsymbol{z}, \quad R^{c}(S) \in S O(3, C)=S L(2, C) / Z_{2} .
\end{aligned}
$$

Here we made use of the fact that the trace and the determinant of a matrix are invariant under conjugation, and the fact that $\operatorname{det} \boldsymbol{z} \cdot \boldsymbol{\sigma}=-\boldsymbol{z} \cdot \boldsymbol{z}$. The superscript over $R$ is to remind us that we are dealing with the complex, rather than the real, orthogonal group, and $z$ is to be viewed as a column vector. 
For any Lie group $G$ with centre $\Omega$, the adjoint representation is a faithful representation of the quotient group $G / \Omega$. In the cases of $S U(2)$ and $S U(1,1)$ we have seen that the adjoint representations coincide respectively with the defining representations of $S O(3)$ and $S O(2,1)$, consistent with the fact that $S U(2) / Z_{2}=S O(3), \quad S U(1,1) / Z_{2}=S O(2,1)$. In the case of $S L(2, C)$ we find that the adjoint representation coincides with the defining representation of the complex orthogonal group $S O(3, C)$, with the complex $3 \times 3$ matrices of the latter rewritten as real $6 \times 6$ matrices in a natural manner.

The group $S L(2, C)$ is related also to the Lorentz group $S O(3,1)$ in a well known two-to-one manner. To see this, consider a generic hermitian matrix

$$
H=\xi_{0}+\boldsymbol{\xi} \cdot \boldsymbol{\sigma}
$$

where $\xi_{0}$ is a real scalar and $\boldsymbol{\xi} \in \mathcal{R}^{3}$. We have $\operatorname{tr} H=2 \xi_{0}$ and $\operatorname{det} H=\xi_{0}{ }^{2}-\boldsymbol{\xi} \cdot \boldsymbol{\xi}$. Clearly, for any $S \in S L(2, C)$, the map $S: H \rightarrow S H S^{\dagger}$ preserves $\operatorname{det} H$, hermiticity of $H$, and the signature of $\operatorname{tr} H$ (the numerical value of $\operatorname{tr} H$ is not preserved since the map is a congruence and not conjugation):

$$
\begin{aligned}
S: \xi_{0}+\boldsymbol{\xi} \cdot \boldsymbol{\sigma} & \rightarrow S\left(\xi_{0}+\boldsymbol{\xi} \cdot \boldsymbol{\sigma}\right) S^{\dagger}=\xi_{0}^{\prime}+\boldsymbol{\xi}^{\prime} \cdot \boldsymbol{\sigma}, \\
\xi_{0}^{\prime 2}-\boldsymbol{\xi}^{\prime} \cdot \boldsymbol{\xi}^{\prime} & =\xi_{0}^{2}-\boldsymbol{\xi} \cdot \boldsymbol{\xi} \\
\operatorname{sign} \xi_{0}^{\prime} & =\operatorname{sign} \xi_{0} .
\end{aligned}
$$

This implies that $\left(\xi_{0}^{\prime}, \boldsymbol{\xi}^{\prime}\right)$ and $\left(\xi_{0}, \boldsymbol{\xi}\right)$ are related by a real $4 \times 4$ Lorentz transformation matrix $L(S)$ :

$$
\left(\begin{array}{c}
\xi_{0}^{\prime} \\
\boldsymbol{\xi}^{\prime}
\end{array}\right)=L(S)\left(\begin{array}{c}
\xi_{0} \\
\boldsymbol{\xi}
\end{array}\right), \quad L(S) \in S O(3,1) .
$$

Since $Z_{2}=\{ \pm 1\}$ is the kernel of the map (3.5), one concludes that the proper orthochronous Lorentz group is isomorphic to $S L(2, C) / Z_{2}$ :

$$
S O(3,1) \sim S L(2, C) / Z_{2} \sim S O(3, C) .
$$

We may illustrate the connections between these three groups by considering some one parameter subgroups (OPS's). The compact OPS $\exp \left(-i \frac{\theta}{2} \sigma_{1}\right)$ generated by (the hermitian) $\sigma_{1}$ takes the following forms

$$
\begin{aligned}
g(\theta) & =\left(\begin{array}{cc}
\cos (\theta / 2) & -i \sin (\theta / 2) \\
-i \sin (\theta / 2) & \cos (\theta / 2)
\end{array}\right), \quad 0 \leq \theta<4 \pi \\
R^{c}(\theta) & =\left(\begin{array}{ccc}
1 & 0 & 0 \\
0 & \cos \theta & -\sin \theta \\
0 & \sin \theta & \cos \theta
\end{array}\right), \quad 0 \leq \theta<2 \pi \\
\Lambda(\theta) & =\left(\begin{array}{cccc}
1 & 0 & 0 & 0 \\
0 & 1 & 0 & 0 \\
0 & 0 & \cos \theta & -\sin \theta \\
0 & 0 & \sin \theta & \cos \theta
\end{array}\right), \quad 0 \leq \theta<2 \pi
\end{aligned}
$$

respectively in $S L(2, C), S O(3, C)$ and $S O(3,1)$. On the other hand, the noncompact OPS $\exp \left(\frac{\beta}{2} \sigma_{1}\right)$ generated by (the antihermitian) $i \sigma_{1}$ takes the following forms:

$$
\begin{aligned}
g(\beta)= & \left(\begin{array}{cc}
\cosh (\beta / 2) & \sinh (\beta / 2) \\
\sinh (\beta / 2) & \cosh (\beta / 2)
\end{array}\right), \quad \beta \in \mathcal{R} ; \\
R^{c}(\beta) & =\left(\begin{array}{ccc}
1 & 0 & 0 \\
0 & \cosh \beta & -i \sinh \beta \\
0 & i \sinh \beta & \cosh \beta
\end{array}\right), \quad \beta \in \mathcal{R} \\
\Lambda(\beta) & =\left(\begin{array}{cccc}
\cosh \beta & \sinh \beta & 0 & 0 \\
\sinh \beta & \cosh \beta & 0 & 0 \\
0 & 0 & 1 & 0 \\
0 & 0 & 0 & 1
\end{array}\right), \quad \beta \in \mathcal{R}
\end{aligned}
$$

It is the identification of $\theta$ and $\theta+2 \pi$ for compact OPS's in going over from $S L(2, C)$ to $S O(3, C)$ or $S O(3,1)$ that is ultimately responsible for the fact that the latter groups are topologically different from the former. 
The adjoint orbits $[12,11]$ of the Lie algebra of $S L(2, C)$ can be easily figured out from the fact that $\boldsymbol{z} \cdot \boldsymbol{z}$ is the only (complex) invariant associated with a generic Lie algebra element $\boldsymbol{z} \cdot \boldsymbol{\sigma}$ under the adjoint action. Since $\boldsymbol{z} \cdot \boldsymbol{z}$ can assume any numerical value, it is clear that there exists precisely one orbit for each point in the complex plane. Closer examination shows that these orbits fall into two different types $[12,11]$.

type-I orbits: $\boldsymbol{z} \cdot \boldsymbol{z}=r^{2} \mathrm{e}^{2 i \phi}, 0 \leq \phi<\pi, 0<r<\infty$.

In this case the Lie algebra element $\boldsymbol{z} \cdot \boldsymbol{\sigma}$ with $\boldsymbol{z} \cdot \boldsymbol{z} \neq 0$ can be brought, using the adjoint action, into the canonical form

$$
\begin{aligned}
\boldsymbol{z}=\left(z_{1}, z_{2}, z_{3}\right) & \rightarrow \boldsymbol{z}_{0}=\left(r \mathrm{e}^{i \phi}, 0,0\right), \\
\boldsymbol{z} \cdot \boldsymbol{\sigma} & \rightarrow \boldsymbol{z}_{0} \cdot \boldsymbol{\sigma}=\operatorname{re}^{i \phi} \sigma_{1} .
\end{aligned}
$$

Clearly, the stability group of this canonical form is

$$
\begin{aligned}
G_{I} & =S O(2) \times S O(1,1)=S O(2, C) \\
& =\left\{\exp \left(-\frac{i}{2}(\alpha+i \beta) \sigma_{1}\right), \quad 0 \leq \alpha<4 \pi, \quad \beta \in \mathcal{R}\right\},
\end{aligned}
$$

which has the topology of the cylinder $\mathcal{R} \times S^{1}$. Thus the type-I orbits have the structure of the four dimensional manifold $S O(3, C) / G_{I}$, and there exists precisely one such orbit for every nonzero complex number.

type-II orbit: $z \cdot z=0$.

A Lie algebra element $\boldsymbol{z} \cdot \boldsymbol{\sigma}$ with $\boldsymbol{z} \cdot \boldsymbol{z}=0$ can be brought, using the adjoint action, into the canonical form

$$
\begin{aligned}
\boldsymbol{z}=\left(z_{1}, z_{2}, z_{3}\right) & \rightarrow \boldsymbol{z}_{0}=(1, i, 0) \\
\boldsymbol{z} \cdot \boldsymbol{\sigma} & \rightarrow \boldsymbol{z}_{0} \cdot \boldsymbol{\sigma}=\sigma_{1}+i \sigma_{2} .
\end{aligned}
$$

The stability group of this canonical form is

$$
G_{I I}=\left\{\exp \left(-\frac{i}{2}(\alpha+i \beta)\left(\sigma_{1}+i \sigma_{2}\right)\right), \quad \alpha, \beta \in \mathcal{R}\right\},
$$

which has the topology of $\mathcal{R}^{2}$. Thus the type-II orbit has the structure of the four dimensional manifold $S O(3, C) / G_{I I}$. Both $G_{I}$ and $G_{I I}$ are abelian. At the risk of repetition we wish to note that there exists only one type-II orbit.

To construct turns for $S L(2, C)$ we shall make use of the orbit $\boldsymbol{z} \cdot \boldsymbol{z}=1$. We shall denote this orbit by $\Sigma$, and refer to it as the orbit of complex unit vectors. For convenience, elements of $\Sigma$ will be decorated with a hat, like $\widehat{\boldsymbol{x}}, \widehat{\boldsymbol{y}}, \ldots$

To conclude this Section, we note that the vector algebra in $\mathcal{R}^{3}$ carries over to $C^{3}$ with virtually no change. In particular, the following familiar identities are true for complex-valued vectors as well:

$$
\begin{aligned}
& \boldsymbol{a} \wedge(\boldsymbol{b} \wedge \boldsymbol{c})=\boldsymbol{b}(\boldsymbol{a} \cdot \boldsymbol{c})-\boldsymbol{c}(\boldsymbol{a} \cdot \boldsymbol{b}) \\
&(\boldsymbol{a} \wedge \boldsymbol{b}) \cdot(\boldsymbol{c} \wedge \boldsymbol{d})=(\boldsymbol{a} \cdot \boldsymbol{c})(\boldsymbol{b} \cdot \boldsymbol{d})-(\boldsymbol{a} \cdot \boldsymbol{d})(\boldsymbol{b} \cdot \boldsymbol{c}) \\
&(\boldsymbol{z} \cdot \boldsymbol{y})(\boldsymbol{x} \cdot \boldsymbol{z})-(\boldsymbol{z} \wedge \boldsymbol{y}) \cdot(\boldsymbol{x} \wedge \boldsymbol{z})=(\boldsymbol{z} \cdot \boldsymbol{z})(\boldsymbol{x} \cdot \boldsymbol{y}), \\
&(\boldsymbol{z} \cdot \boldsymbol{z})(\boldsymbol{x} \wedge \boldsymbol{y})=(\boldsymbol{z} \cdot \boldsymbol{y})(\boldsymbol{x} \wedge \boldsymbol{z})+(\boldsymbol{z} \cdot \boldsymbol{x})(\boldsymbol{z} \wedge \boldsymbol{y}) \\
&+(\boldsymbol{z} \wedge \boldsymbol{y}) \wedge(\boldsymbol{x} \wedge \boldsymbol{z}) .
\end{aligned}
$$

These relations will be found useful in the next Section.

\section{CONSTRUCTION OF TURNS FOR $S L(2, C)$}

We now have all the ingredients to construct a theory of turns for $S L(2, C)$. Elements of $S L(2, C)$ can be described through

$$
S\left(a_{0}, \boldsymbol{a}\right)=a_{0}-i \boldsymbol{a} \cdot \boldsymbol{\sigma},
$$


where $a_{0}$ is a complex scalar and $\boldsymbol{a}$ a complex three vector subject to the condition $a_{0}^{2}+\boldsymbol{a} \cdot \boldsymbol{a}=1$. This is one complex condition on four complex numbers. Given $S\left(a_{0}, \boldsymbol{a}\right) \in S L(2, C)$, to construct the turn corresponding to $S\left(a_{0}, \boldsymbol{a}\right)$ we look for pairs of complex unit vectors $\widehat{\boldsymbol{x}}, \widehat{\boldsymbol{y}}$ such that $a_{0}=\widehat{\boldsymbol{x}} \cdot \widehat{\boldsymbol{y}}$ and $\boldsymbol{a}=\widehat{\boldsymbol{x}} \wedge \widehat{\boldsymbol{y}}$.

Let $\widehat{\boldsymbol{x}}$ be a unit vector perpendicular to $\boldsymbol{a}$, i.e. $\widehat{\boldsymbol{x}} \cdot \boldsymbol{a}=0$. This corresponds to two real constraints in the fourparameter manifold of unit vectors. Thus, there is a two parameter worth of freedom in the choice of $\widehat{\boldsymbol{x}}$ perpendicular to $\boldsymbol{a}$. Define

$$
\boldsymbol{y} \equiv a_{0} \widehat{\boldsymbol{x}}+\boldsymbol{a} \wedge \widehat{\boldsymbol{x}} .
$$

Then $a_{0}^{2}+\boldsymbol{a} \cdot \boldsymbol{a}=1$ guarantees that $\boldsymbol{y} \cdot \boldsymbol{y}=1$, and we may indeed write $\widehat{\boldsymbol{y}}$ in place of $\boldsymbol{y}$. Further, it can be verified that the conditions $a_{0}=\widehat{\boldsymbol{x}} \cdot \widehat{\boldsymbol{y}}$ and $\boldsymbol{a}=\widehat{\boldsymbol{x}} \wedge \widehat{\boldsymbol{y}}$ are satisfied. Since there is a two-parameter worth of freedom in the choice of $\widehat{\boldsymbol{x}}$, we have not just one pair but an equivalence class of pairs $\widehat{\boldsymbol{x}}, \widehat{\boldsymbol{y}}$ representing a given $S \in S L(2, C)$. We may use any one of these pairs and write

$$
\begin{aligned}
S\left(a_{0}, \boldsymbol{a}\right) & =a_{0}-i \boldsymbol{a} . \boldsymbol{\sigma} \\
& =\widehat{\boldsymbol{x}} \cdot \widehat{\boldsymbol{y}}-i \widehat{\boldsymbol{x}} \wedge \widehat{\boldsymbol{y}} \cdot \boldsymbol{\sigma} \equiv S(\widehat{\boldsymbol{x}}, \widehat{\boldsymbol{y}}),
\end{aligned}
$$

and call the equivalence class $S(\widehat{\boldsymbol{x}}, \widehat{\boldsymbol{y}})$ as the turn from the tail $\widehat{\boldsymbol{x}}$ to the head $\widehat{\boldsymbol{y}}$. (The improper use of a common symbol $S(\cdot, \cdot)$ at the start and the end of this equation should cause no confusion.) Conversely, for any choice of two complex unit vectors $\widehat{\boldsymbol{x}}$ and $\widehat{\boldsymbol{y}}, S(\widehat{\boldsymbol{x}}, \widehat{\boldsymbol{y}})$ is indeed an $S L(2, C)$ element, and two pairs $\widehat{\boldsymbol{x}}, \widehat{\boldsymbol{y}}$ and $\widehat{\boldsymbol{x}}^{\prime}, \widehat{\boldsymbol{y}}^{\prime}$ are in the same equivalence class if and only if $\widehat{\boldsymbol{x}} \cdot \widehat{\boldsymbol{y}}=\widehat{\boldsymbol{x}}^{\prime} \cdot \widehat{\boldsymbol{y}}^{\prime}$ and $\widehat{\boldsymbol{x}} \wedge \widehat{\boldsymbol{y}}=\widehat{\boldsymbol{x}}^{\prime} \wedge \widehat{\boldsymbol{y}}^{\prime}$. For the special elements $\pm 1 \in S L(2, C)$, we have $\boldsymbol{a}=0$ and hence $\widehat{\boldsymbol{y}}= \pm \widehat{\boldsymbol{x}}$.

Since $S\left(a_{0}, \boldsymbol{a}\right)^{-1}=S\left(a_{0},-\boldsymbol{a}\right)$, it follows that

$$
S(\widehat{\boldsymbol{x}}, \widehat{\boldsymbol{y}})^{-1}=S(\widehat{\boldsymbol{y}}, \widehat{\boldsymbol{x}}) .
$$

Thus the inverse corresponds to reversing the sense of the turn, as one would have wished. Only the parallelogram law of composition remains to be established.

With the aid of the identities (3.14), it is readily seen that the definition (4.3) possesses the following property:

$$
S(\widehat{\boldsymbol{z}}, \widehat{\boldsymbol{y}}) S(\widehat{\boldsymbol{x}}, \widehat{\boldsymbol{z}})=S(\widehat{\boldsymbol{x}}, \widehat{\boldsymbol{y}}) .
$$

This is the parallelogram law when the head of the right factor coincides with the tail of the left one. The theory of turns for $S L(2, C)$ will be deemed complete if one can show that there always exists a common unit vector $\widehat{z}$ where two turns meet.

Suppose $a_{0}-i \boldsymbol{a} . \boldsymbol{\sigma}$ and $b_{0}-i \boldsymbol{b} . \boldsymbol{\sigma}$ are two $S L(2, C)$ elements, then the common unit vector $\widehat{\boldsymbol{z}}$ where the corresponding turns meet should necessarily satisfy $\boldsymbol{a} . \widehat{\boldsymbol{z}}=0$ and $\boldsymbol{b} . \widehat{\boldsymbol{z}}=0$. Thus $\widehat{\boldsymbol{z}}$ must be a multiple of $\boldsymbol{a} \wedge \boldsymbol{b}$. It follows that a unit vector $\widehat{\boldsymbol{z}}$ common to two turns exists if and only if $(\boldsymbol{a} \wedge \boldsymbol{b}) \cdot(\boldsymbol{a} \wedge \boldsymbol{b}) \neq 0$.

Thus, if the two $S L(2, C)$ elements to be composed are such that $(\boldsymbol{a} \wedge \boldsymbol{b}) \cdot(\boldsymbol{a} \wedge \boldsymbol{b}) \neq 0$, then their turns meet in the space of unit vectors $\Sigma$, and (4.5) constitutes the parallelogram law of composition for such pairs. But if $(\boldsymbol{a} \wedge \boldsymbol{b}) \cdot(\boldsymbol{a} \wedge \boldsymbol{b})=0$, the corresponding turns do not meet in $\Sigma$, and hence further work is needed before (4.5) can be used as the parallelogram law of composition for such pairs. Fortunately, the latter situation occurs only in a measure zero set of cases: $(\boldsymbol{a} \wedge \boldsymbol{b}) \cdot(\boldsymbol{a} \wedge \boldsymbol{b})$ can take any complex value, but the turns fail to meet in $\Sigma$ only when this value is zero.

In such special cases one may infinitesimally modify one of the two $S L(2, C)$ elements to be composed, say change $S\left(b_{0}, \boldsymbol{b}\right)$ to $S\left(b_{0}^{\prime}, \boldsymbol{b}^{\prime}\right)$ so that $b_{0}^{\prime}-b_{0}$ and $\boldsymbol{b}^{\prime}-\boldsymbol{b}$ are infinitesimals and $\left(\boldsymbol{a} \wedge \boldsymbol{b}^{\prime}\right) \cdot\left(\boldsymbol{a} \wedge \boldsymbol{b}^{\prime}\right) \neq 0$, use (4.5) to compose the turns, and take the limit of the composed turn as the infinitesimals go to zero. Alternatively, we may suitably factorize $S\left(b_{0}, \boldsymbol{b}\right)$ into a product of two $S L(2, C)$ elements, $S\left(b_{0}, \boldsymbol{b}\right)=S\left(b_{0}^{\prime \prime}, \boldsymbol{b}^{\prime \prime}\right) S\left(b_{0}^{\prime}, \boldsymbol{b}^{\prime}\right)$, and use the parallelogram law twice: compose the turn corresponding to $S\left(a_{0}, \boldsymbol{a}\right)$ with that corresponding to $S\left(b_{0}^{\prime}, \boldsymbol{b}^{\prime}\right)$, and then compose the resulting turn with that corresponding to $S\left(b_{0}^{\prime \prime}, \boldsymbol{b}^{\prime \prime}\right)$ (this latter approach is the one used in the theory of turns for $S U(1,1)$ presented in Ref. [3,4] for handling turns which do not meet).

With these provisions for handling the measure zero case of nonintersecting turns, (4.5) constitutes the parallelogram law of composition for $S L(2, C)$ turns.

\section{TURNS AND THE POLAR DECOMPOSITION}

In this Section we describe in the language of turns we have just developed the process of polar decomposition. Such an exercise not only helps us in developing a feel for $S L(2, C)$ turns, but also helps in the study of composition of Lorentz boosts to be taken up in the next Section. 
Any element $S \in S L(2, C)$ can be decomposed as $S=H U$, where $H$ is hermitian positive definite, $U$ is unitary, and therefore $H, U \in S L(2, C)$. This polar decomposition is unique for a given $S$, and corresponds to the decomposition of an element $\Lambda \in S O(3,1)$ into a spatial rotation followed by a boost. That is, $\Lambda=P R$ where $P$ is real symmetric positive definite and $R$ is orthogonal. Again, $P$ and $R$ are uniquely determined in terms of $\Lambda$, and both factors are elements of $S O(3,1)$. In $S O(3, C)$, this decomposition corresponds to a rotation through a purely real angle followed by a rotation through a purely imaginary angle. It is important to appreciate that polar decomposition is not covariant under conjugation by $S L(2, C)$ [equivalently, by $S O(3,1)$ or $S O(3, C)$ ]. It is covariant only under $S U(2)$ [equivalently, under $S O(3)]$.

Now an element $a_{0}-i \boldsymbol{a} . \boldsymbol{\sigma} \in S L(2, C)$ is unitary (real rotation) if and only if $a_{0}$ and $\boldsymbol{a}$ are real. In the language of turns this translates into the following statement: $S=\widehat{\boldsymbol{x}} \cdot \widehat{\boldsymbol{y}}-i(\widehat{\boldsymbol{x}} \wedge \widehat{\boldsymbol{y}}) \cdot \boldsymbol{\sigma}$ is unitary if and only if $\widehat{\boldsymbol{x}}$ and $\widehat{\boldsymbol{y}}$ are real. Similarly $S=a_{0}-i \boldsymbol{a} . \boldsymbol{\sigma}$ is hermitian positive definite (pure boost) if and only if $a_{0}$ is real and $>0$ and $\boldsymbol{a}$ is imaginary. In terms of turns this happens if one member of the pair $(\widehat{\boldsymbol{x}}, \widehat{\boldsymbol{y}})$, say $\widehat{\boldsymbol{x}}$, is purely real to which the real part of the other, say $\widehat{\boldsymbol{y}}$, is parallel (not antiparallel). The imaginary part of $\widehat{\boldsymbol{y}}$ is then perpendicular to $\widehat{\boldsymbol{x}}$ by virtue of the fact that the real and imaginary parts of a complex unit vector are necessarily orthogonal.

Writing $\boldsymbol{a}=\boldsymbol{a}_{R}+i \boldsymbol{a}_{I}$, polar decomposition of $S\left(a_{0}, \boldsymbol{a}\right)=a_{0}-i \boldsymbol{a} . \boldsymbol{\sigma}$ is trivial when $\boldsymbol{a}_{R}$ and $\boldsymbol{a}_{I}$, the real and imaginary parts of $\boldsymbol{a}$, are multiples of one another. So we assume that $\boldsymbol{a}_{R}$ and $\boldsymbol{a}_{I}$ are linearly independent. Our aim is to decompose $S(\widehat{\boldsymbol{x}}, \widehat{\boldsymbol{y}}) \in S L(2, C)$ in the polar form

$$
\begin{aligned}
S & =a_{0}-i \boldsymbol{a} . \boldsymbol{\sigma} \\
& =S(\widehat{\boldsymbol{x}}, \widehat{\boldsymbol{y}})=S(\widehat{\boldsymbol{z}}, \widehat{\boldsymbol{y}}) S(\widehat{\boldsymbol{x}}, \widehat{\boldsymbol{z}}),
\end{aligned}
$$

with $\widehat{\boldsymbol{x}}, \widehat{\boldsymbol{z}}$ real so that $S(\widehat{\boldsymbol{x}}, \widehat{\boldsymbol{z}})$ will be an element of $S U(2)$ (i.e., rotation) and the real part of $\widehat{\boldsymbol{y}}$ parallel to $\widehat{\boldsymbol{z}}$ so that $S(\widehat{\boldsymbol{z}}, \widehat{\boldsymbol{y}})$ will be hermitian positive definite (i.e., pure boost).

We noted in Section IV that there exists a two parameter worth of freedom in choosing the tail point $\widehat{\boldsymbol{x}}$ of a turn $S(\widehat{\boldsymbol{x}}, \widehat{\boldsymbol{y}})$. To facilitate polar decomposition we need to choose $\widehat{\boldsymbol{x}}$ to be real, and the above two parameter freedom permits such a choice. Indeed, reality of $\widehat{\boldsymbol{x}}$ and the requirement $\boldsymbol{a} \cdot \widehat{\boldsymbol{x}}=0$, which follows from $\boldsymbol{a}=\widehat{\boldsymbol{x}} \wedge \widehat{\boldsymbol{y}}$, together imply that $\widehat{\boldsymbol{x}}$ is necessarily orthogonal to both the real and imaginary parts of $\boldsymbol{a}=\boldsymbol{a}_{R}+i \boldsymbol{a}_{I}$ :

$$
\widehat{\boldsymbol{x}}= \pm \frac{\boldsymbol{a}_{R} \wedge \boldsymbol{a}_{I}}{\sqrt{\left(\boldsymbol{a}_{R} \wedge \boldsymbol{a}_{I}\right) \cdot\left(\boldsymbol{a}_{R} \wedge \boldsymbol{a}_{I}\right)}} .
$$

Then, from (4.2),

$$
\begin{aligned}
\widehat{\boldsymbol{y}} & =a_{0} \widehat{\boldsymbol{x}}+\boldsymbol{a} \wedge \widehat{\boldsymbol{x}} \\
& =a_{0 R} \widehat{\boldsymbol{x}}+\boldsymbol{a}_{R} \wedge \widehat{\boldsymbol{x}}+i a_{0 I} \widehat{\boldsymbol{x}}+i \boldsymbol{a}_{I} \wedge \widehat{\boldsymbol{x}},
\end{aligned}
$$

where $a_{0 R}$ and $a_{0 I}$ are respectively the real and imaginary parts of the scalar $a_{0}$. Since $\widehat{\boldsymbol{z}}$ has to be real and parallel ( not antiparallel) to the real part of $\widehat{\boldsymbol{y}}$, we have

$$
\widehat{\boldsymbol{z}}=\frac{a_{0 R} \widehat{\boldsymbol{x}}+\boldsymbol{a}_{R} \wedge \widehat{\boldsymbol{x}}}{\sqrt{a_{0 R}^{2}+\boldsymbol{a}_{R} \cdot \boldsymbol{a}_{R}}},
$$

and the polar decomposition is completed.

Reality of $\widehat{\boldsymbol{x}}$ required by polar decomposition removed the two parameter worth of arbitrariness or freedom in the choice of $\widehat{\boldsymbol{x}}$, and hence in that of $\widehat{\boldsymbol{z}}$ and $\widehat{\boldsymbol{y}}$, except for a signature in (5.2). Since $\widehat{\boldsymbol{y}}$ and $\widehat{\boldsymbol{z}}$ in (5.3), (5.4) are linear in $\widehat{\boldsymbol{x}}$, and since the quantities entering the polar decomposition are quadratic in $\widehat{\boldsymbol{x}}, \widehat{\boldsymbol{y}}$ and $\widehat{\boldsymbol{z}}$, either choice for the signature in (5.2) leads to the same set of expressions, confirming the uniqueness of the polar decomposition:

$$
\begin{aligned}
S\left(a_{0}, \boldsymbol{a}\right) & =a_{0}-i \boldsymbol{a} \cdot \boldsymbol{\sigma} \\
& =S(\widehat{\boldsymbol{z}}, \widehat{\boldsymbol{y}}) S(\widehat{\boldsymbol{x}}, \widehat{\boldsymbol{z}}) \\
& =(\widehat{\boldsymbol{z}} \cdot \widehat{\boldsymbol{y}}-i \widehat{\boldsymbol{z}} \wedge \widehat{\boldsymbol{y}} \cdot \boldsymbol{\sigma})(\widehat{\boldsymbol{x}} \cdot \widehat{\boldsymbol{z}}-i \widehat{\boldsymbol{x}} \wedge \widehat{\boldsymbol{z}} \cdot \boldsymbol{\sigma}) ; \\
\widehat{\boldsymbol{x}} \cdot \widehat{\boldsymbol{z}} & =\frac{a_{0 R}}{\sqrt{\left(a_{0 R}\right)^{2}+\boldsymbol{a}_{R} \cdot \boldsymbol{a}_{R}}}, \\
\widehat{\boldsymbol{x}} \wedge \widehat{\boldsymbol{z}} & =\frac{\boldsymbol{a}_{R}}{\sqrt{\left(a_{0 R}\right)^{2}+\boldsymbol{a}_{R} \cdot \boldsymbol{a}_{R}}} \\
\widehat{\boldsymbol{z}} \cdot \widehat{\boldsymbol{y}} & =\sqrt{\left(a_{0 R}\right)^{2}+\boldsymbol{a}_{R} \cdot \boldsymbol{a}_{R}}>1, \\
\widehat{\boldsymbol{z}} \wedge \widehat{\boldsymbol{y}} & =\frac{i\left(a_{R} \boldsymbol{a}_{I}-a_{0 I} \boldsymbol{a}_{R}+\boldsymbol{a}_{R} \wedge \boldsymbol{a}_{I}\right)}{\sqrt{\left(a_{0 R}\right)^{2}+\boldsymbol{a}_{R} \cdot \boldsymbol{a}_{R}}} .
\end{aligned}
$$


We have $\widehat{\boldsymbol{z}} \cdot \widehat{\boldsymbol{y}}>1$ by virtue of the fact that for a complex unit vector the square of the norm of the real part exceeds unity by an amount equal to the square of the norm of the imaginary part.

Let us define a real angle $\epsilon$ and real unit vector $\widehat{\boldsymbol{k}}_{r}$ through

$$
\begin{aligned}
\widehat{\boldsymbol{x}} \cdot \widehat{\boldsymbol{z}} & =\cos (\epsilon / 2), \\
\widehat{\boldsymbol{x}} \wedge \widehat{\boldsymbol{z}} & =\sin (\epsilon / 2) \widehat{\boldsymbol{k}}_{r} .
\end{aligned}
$$

These expressions remain invariant under the transformation $\epsilon / 2 \rightarrow-\epsilon / 2, \widehat{\boldsymbol{k}}_{r} \rightarrow-\widehat{\boldsymbol{k}}_{r}$, and hence we restrict the range of $\epsilon$ to $0 \leq \epsilon / 2 \leq \pi$. Similarly, let us define a real positive rapidity parameter $\beta$ and a real unit vector $\widehat{\boldsymbol{k}}_{b}$ through

$$
\begin{aligned}
\widehat{\boldsymbol{z}} \cdot \widehat{\boldsymbol{y}} & =\cosh (\beta / 2), \\
\widehat{\boldsymbol{z}} \wedge \widehat{\boldsymbol{y}} & =i \sinh (\beta / 2) \widehat{\boldsymbol{k}}_{b} .
\end{aligned}
$$

With these definitions the polar decomposition (3.5) can be written as

$$
\begin{aligned}
S\left(a_{0}, \boldsymbol{a}\right) & =a_{0}-i \boldsymbol{a} \cdot \boldsymbol{\sigma} \\
& =\left(\cosh (\beta / 2)+\sinh (\beta / 2) \widehat{\boldsymbol{k}}_{b} \cdot \boldsymbol{\sigma}\right)\left(\cos (\epsilon / 2)-i \sin (\epsilon / 2) \widehat{\boldsymbol{k}}_{r} \cdot \boldsymbol{\sigma}\right) .
\end{aligned}
$$

The hyperbolic factor is manifestly hermitian positive definite with eigenvalues $\exp ( \pm \beta / 2)$, and corresponds to a boost along the spatial direction $\widehat{\boldsymbol{k}}_{b}$ with rapidity parameter $\beta$. The trigonometric factor is manifestly unitary and corresponds to spatial rotation by angle $\epsilon$ about the direction $\widehat{\boldsymbol{k}}_{r}$. The suffix $b / r$ attached to $\widehat{\boldsymbol{k}}$ signifies boost/rotation. Comparing (5.6), (5.7) with (5.5) we deduce that

$$
\begin{aligned}
\cosh (\beta / 2) & =\sqrt{\left(a_{0 R}\right)^{2}+\boldsymbol{a}_{R} \cdot \boldsymbol{a}_{R}}, \\
\tan (\epsilon / 2) & =\frac{\sqrt{\boldsymbol{a}_{R} \cdot \boldsymbol{a}_{R}}}{a_{0 R}},
\end{aligned}
$$

and that the spatial rotation is about the direction of $\left(\operatorname{sign} a_{0 R}\right) \boldsymbol{a}_{R}$, while the boost is along the spatial direction $\left(a_{R} \boldsymbol{a}_{I}-a_{0 I} \boldsymbol{a}_{R}+\boldsymbol{a}_{R} \wedge \boldsymbol{a}_{I}\right)$.

In concluding this Section we wish to make the following remark. Since the set of all $2 \times 2$ hermitian positive definite $S L(2, C)$ matrices $\exp (\boldsymbol{x} . \boldsymbol{\sigma}), \boldsymbol{x} \in \mathcal{R}^{3}$ has the structure of $\mathcal{R}^{3}$ as a manifold, and since $S U(2)$ has the structure of $S^{3}$, it follows from the uniqueness of the polar decomposition that the $S L(2, C)$ group manifold has the structure of $\mathcal{R}^{3} \times S^{3}$. In the case of $S O(3,1)[S O(3, C)]$, the role of $S^{3}$ will be played by the real projective space $R P^{3}=S^{3} / Z_{2}$, consistent with the relation $S O(3,1) \sim S O(3, C)=S L(2, C) / Z_{2}$.

\section{COMPOSITION OF BOOSTS: WIGNER OR THOMAS ROTATION}

As our second and final illustration of the theory of turns we have developed for the Lorentz group, we apply it to the well known problem of composition of Lorentz boosts. Let the first boost be in the spatial direction of the real unit vecor $\widehat{\boldsymbol{m}}$, with rapidity parameter $\beta_{m}$, and let the second be in the direction of $\widehat{\boldsymbol{n}}$, with rapidity parameter $\beta_{n}$. The problem of composition of boosts is trivial when $\widehat{\boldsymbol{m}}$ and $\widehat{\boldsymbol{n}}$ are parallel or antiparallel to one another, and so we assume $\widehat{\boldsymbol{m}}$ and $\widehat{\boldsymbol{n}}$ to be linearly independent. Let $\left(\widehat{\boldsymbol{x}}_{m}, \widehat{\boldsymbol{y}}_{m}\right)$ and $\left(\widehat{\boldsymbol{x}}_{n}, \widehat{\boldsymbol{y}}_{n}\right)$ be the turns corresponding to the two boosts. Then

$$
\begin{aligned}
\widehat{\boldsymbol{x}}_{m} \cdot \widehat{\boldsymbol{y}}_{m} & =\cosh \left(\beta_{m} / 2\right), \\
\widehat{\boldsymbol{x}}_{m} \wedge \widehat{\boldsymbol{y}}_{m} & =i \sinh \left(\beta_{m} / 2\right) \widehat{\boldsymbol{m}} \\
\widehat{\boldsymbol{x}}_{n} \cdot \widehat{\boldsymbol{y}}_{n} & =\cosh \left(\beta_{n} / 2\right), \\
\widehat{\boldsymbol{x}}_{n} \wedge \widehat{\boldsymbol{y}}_{n} & =i \sinh \left(\beta_{n} / 2\right) \widehat{\boldsymbol{n}} .
\end{aligned}
$$

Let $\widehat{z}$ be the point where the head of the first turn and the tail of the second turn meet (it turns out that $\widehat{z}$ is necessarily real). Since the pair $\left(\widehat{\boldsymbol{x}}_{m}, \widehat{\boldsymbol{y}}_{m}\right)$ is orthogonal to $\widehat{\boldsymbol{m}}$ while the pair $\left(\widehat{\boldsymbol{x}}_{n}, \widehat{\boldsymbol{y}}_{n}\right)$ is orthogonal to $\widehat{\boldsymbol{n}}$, the common meeting point $\widehat{\boldsymbol{z}}$ has to be necessarily orthogonal to both $\widehat{\boldsymbol{m}}$ and $\widehat{\boldsymbol{n}}$ :

$$
\widehat{z}= \pm \frac{\widehat{m} \wedge \widehat{n}}{\sqrt{(\widehat{m} \wedge \widehat{n}) \cdot(\widehat{m} \wedge \widehat{n})}}
$$


Let $\widehat{\boldsymbol{x}}$ be the tail of the first turn and $\widehat{\boldsymbol{y}}$ be the head of the second turn when they so meet at $\widehat{\boldsymbol{z}}$, so that the first boost can be represented by $(\widehat{\boldsymbol{x}}, \widehat{\boldsymbol{z}})$ and the second by $(\widehat{\boldsymbol{z}}, \widehat{\boldsymbol{y}})$. That is, $S\left(\widehat{\boldsymbol{x}}_{m}, \widehat{\boldsymbol{y}}_{m}\right)=S(\widehat{\boldsymbol{x}}, \widehat{\boldsymbol{z}})$ and $S\left(\widehat{\boldsymbol{x}}_{n}, \widehat{\boldsymbol{y}}_{n}\right)=S(\widehat{\boldsymbol{z}}, \widehat{\boldsymbol{y}})$. Solving for $\widehat{\boldsymbol{x}}, \widehat{\boldsymbol{y}}$ we have,

$$
\begin{aligned}
& \widehat{\boldsymbol{x}}=\cosh \left(\beta_{m} / 2\right) \widehat{\boldsymbol{z}}+i \sinh \left(\beta_{m} / 2\right) \widehat{\boldsymbol{z}} \wedge \widehat{\boldsymbol{m}} \\
& \widehat{\boldsymbol{y}}=\cosh \left(\beta_{n} / 2\right) \widehat{\boldsymbol{z}}+i \sinh \left(\beta_{n} / 2\right) \widehat{\boldsymbol{n}} \wedge \widehat{\boldsymbol{z}}
\end{aligned}
$$

We conclude from the parallelogram law (4.5) that the product of the $\widehat{\boldsymbol{m}}$ boost followed by the $\widehat{\boldsymbol{n}}$ boost is

$$
S\left(\widehat{\boldsymbol{x}}_{n}, \widehat{\boldsymbol{y}}_{n}\right) S\left(\widehat{\boldsymbol{x}}_{m}, \widehat{\boldsymbol{y}}_{m}\right)=S(\widehat{\boldsymbol{z}}, \widehat{\boldsymbol{y}}) S(\widehat{\boldsymbol{x}}, \widehat{\boldsymbol{z}})=S(\widehat{\boldsymbol{x}}, \widehat{\boldsymbol{y}}) .
$$

To gain insight into the product $S(\widehat{\boldsymbol{x}}, \widehat{\boldsymbol{y}}) \in S L(2, C)$, let us write it in the form $S(\widehat{\boldsymbol{x}}, \widehat{\boldsymbol{y}})=a_{0}-i \boldsymbol{a} . \boldsymbol{\sigma}$, where

$$
\begin{aligned}
& a_{0}=\widehat{\boldsymbol{x}} \cdot \widehat{\boldsymbol{y}} \equiv a_{0 R}+i a_{0 I}, \\
& \boldsymbol{a}=\widehat{\boldsymbol{x}} \wedge \widehat{\boldsymbol{y}} \equiv \boldsymbol{a}_{R}+i \boldsymbol{a}_{I} .
\end{aligned}
$$

Use of (6.3) yields the following expressions for the real and imaginary parts of $a_{0}, \boldsymbol{a}$ :

$$
\begin{aligned}
a_{0 R}= & \cosh \left(\beta_{m} / 2\right) \cosh \left(\beta_{n} / 2\right) \\
& \quad+\sinh \left(\beta_{m} / 2\right) \sinh \left(\beta_{n} / 2\right) \widehat{\boldsymbol{m}} \cdot \widehat{\boldsymbol{n}}, \\
a_{0 I}= & 0 ; \\
\boldsymbol{a}_{R}= & \sinh \left(\beta_{m} / 2\right) \sinh \left(\beta_{n} / 2\right) \widehat{\boldsymbol{m}} \wedge \widehat{\boldsymbol{n}}, \\
\boldsymbol{a}_{I}= & \sinh \left(\beta_{m} / 2\right) \cosh \left(\beta_{n} / 2\right) \widehat{\boldsymbol{m}}+\sinh \left(\beta_{n} / 2\right) \cosh \left(\beta_{m} / 2\right) \widehat{\boldsymbol{n}} .
\end{aligned}
$$

The vector parameter $\boldsymbol{a}$ in (6.6) determining the product of the $\widehat{\boldsymbol{m}}$ and $\widehat{\boldsymbol{n}}$ boosts is complex, as was to be expected, since the product of two Lorentz boosts in neither a (pure) boost nor a spatial rotation.

We may now apply the polar decomposition developed in the last Section to the product of the two boosts:

$$
\begin{aligned}
S\left(\widehat{\boldsymbol{x}}_{n}, \widehat{\boldsymbol{y}}_{n}\right) S\left(\widehat{\boldsymbol{x}}_{m}, \widehat{\boldsymbol{y}}_{m}\right) & =a_{0}-i \boldsymbol{a} \cdot \boldsymbol{\sigma} \\
& =S\left(\widehat{\boldsymbol{z}}^{\prime}, \widehat{\boldsymbol{y}}^{\prime}\right) S\left(\widehat{\boldsymbol{x}}^{\prime}, \widehat{\boldsymbol{z}}^{\prime}\right),
\end{aligned}
$$

where we require $S\left(\widehat{\boldsymbol{z}}^{\prime}, \widehat{\boldsymbol{y}}^{\prime}\right)$ to correspond to a boost and $S\left(\widehat{\boldsymbol{x}}^{\prime}, \widehat{\boldsymbol{z}}^{\prime}\right)$ to a spatial rotation, and $a_{0}, \boldsymbol{a}$ are given by (6.6). The spatial rotation so obtained is known as the Wigner or Thomas Rotation [13].

It follows from (5.5) and (6.6) that the Wigner rotation $S\left(\widehat{\boldsymbol{x}}^{\prime}, \widehat{\boldsymbol{z}}^{\prime}\right)$ is about the direction $\boldsymbol{a}_{R} \sim \widehat{\boldsymbol{m}} \wedge \widehat{\boldsymbol{n}}$. That is, the Wigner rotation is in the plane spanned by $\widehat{\boldsymbol{m}}$ and $\widehat{\boldsymbol{n}}$. Let $\epsilon$ be the magnitude of the Wigner rotation. Then from (5.9) we have

$$
\begin{aligned}
\tan (\epsilon / 2) & =\sqrt{\left(a_{0 R}\right)^{2}+\boldsymbol{a}_{R} \cdot \boldsymbol{a}_{R}} \\
& =\frac{\sin \theta}{\kappa+\cos \theta},
\end{aligned}
$$

where $\kappa=\operatorname{coth}\left(\beta_{n} / 2\right) \operatorname{coth}\left(\beta_{m} / 2\right)$, and $\theta$ is the angle between $\widehat{\boldsymbol{m}}$ and $\widehat{\boldsymbol{n}}$.

Having computed the Wigner rotation part, we now examine the boost part $S\left(\widehat{\boldsymbol{z}}^{\prime}, \widehat{\boldsymbol{y}}^{\prime}\right)$. Let $\beta_{\text {res }}$ denote the rapidity parameter for this boost. Then one finds from (5.9)

$$
\begin{aligned}
\cosh \beta_{\text {res }} & =2\left(\left(a_{0 R}\right)^{2}+\boldsymbol{a}_{R} \cdot \boldsymbol{a}_{R}\right)-1 \\
& =\cosh \beta_{m} \cosh \beta_{n}+\sinh \beta_{m} \sinh \beta_{n} \cos \theta .
\end{aligned}
$$

Further, it follows from (5.5) and (6.6) that this resultant boost is in the direction of

$$
\begin{aligned}
-i \widehat{\boldsymbol{z}}^{\prime} \wedge \widehat{\boldsymbol{y}}^{\prime}= & \frac{\left(a_{R} \boldsymbol{a}_{I}-a_{0 I} \boldsymbol{a}_{R}+\boldsymbol{a}_{R} \wedge \boldsymbol{a}_{I}\right)}{\sqrt{\left(a_{0 R}\right)^{2}+\boldsymbol{a}_{R} \cdot \boldsymbol{a}_{R}}} \\
= & \frac{1}{2} \cosh \beta_{m} \sinh \beta_{n} \widehat{\boldsymbol{n}} \\
& +\frac{1}{2} \sinh \beta_{m} \widehat{\boldsymbol{m}}+\sinh ^{2}\left(\beta_{n} / 2\right) \sinh \beta_{m} \cos \theta \widehat{\boldsymbol{n}} .
\end{aligned}
$$


Thus the resultant boost is about a direction in the plane spanned by $\widehat{\boldsymbol{m}}$ and $\widehat{\boldsymbol{n}}$. If $\phi$ is the angle between the resultant boost and $\widehat{\boldsymbol{n}}$, one finds that

$$
\tan \phi=\frac{\sin \theta \sinh \beta_{m}}{\cosh \beta_{m} \sinh \beta_{n}+\cos \theta \cosh \beta_{n} \sinh \beta_{m}} .
$$

Thus the product of two Lorentz boosts of rapidity parameters $\beta_{m}, \beta_{n}$ and spatial directions $\widehat{\boldsymbol{m}}, \widehat{\boldsymbol{n}}$ is a Wigner rotation of amount $\epsilon$ in the plane spanned by $\widehat{\boldsymbol{m}}, \widehat{\boldsymbol{n}}$ followed by a Lorentz boost of rapidity parameter $\beta_{\text {res }}$ along the direction that lies in the plane spanned by $\widehat{\boldsymbol{m}}, \widehat{\boldsymbol{n}}$ and makes an angle $\phi$ with $\widehat{\boldsymbol{n}}$. The expressions for $\epsilon, \beta_{\text {res }}$ and $\phi$ derived using turns are consistent with known results [14], but our aim of this exercise was simply to illustrate the theory of turns we have developed for the Lorentz group.

\section{CONCLUDING REMARKS}

We have examined in detail the ideas underlying Hamilton's theory of turns for $S U(2)$, in a manner that paves the way to developing a similar geometrical construction for other groups. After a brief review of the theories of turns for $S U(2)$ and $S U(1,1)$, we worked out a theory of turns for the group $S L(2, C)$. Formulae for the polar decomposition of an $S L(2, C)$ element were derived within the framework of the theory of turns developed here, and were put to use to compose Lorentz boosts and to calculate the resulting Wigner rotation. These exercises, carried out for illustration of the geometric construction, led to acceptable results. Decomposition of an arbitrary element of the Lorentz group in the form spatial rotation-followed by boost in a fixed direction-followed by spatial rotation can be analysed in a similar manner using turns.

It is gratifying to note that, with our earlier generalization [3,4] of the theory of turns to $S U(1,1) \sim S L(2, R)=$ $S p(2, R)$, the present generalization renders a geometric representation in the spirit of Hamilton available to all low dimensional simple Lie groups of interest in physics.

The geometry of turns for $S U(2)$ is applicable to any problem that involves the group $S U(2)$. Two-level systems constitute an important class of such problems, but not the only ones. As remarked earlier, the theory of turns has led to the formulation and solution of important sythesis problems in these contexts [5-7]. For instance, we have the result that all linear intensity preserving transformations of polarization optics can be synthesised using just two quarterwave plates and one halfwave plate $[7,8]$. Notwithstanding its wording, this result is applicable not only to polarization optics, but also to other systems involving $S U(2)$. For instance, in the case of nuclear magnetic resonance $\pi / 2$ and $\pi$-pulses will correspond respectively to the same $S U(2)$ transformations as quarter and halfwave plates in polarization optics. (These are respectively the eighth and fourth symmetric roots of the unit matrix.) And hence the above result in this context will read: any unitary evolution ( $S U(2)$ transformation ) of a spin half system can be achieved using two $\pi / 2$ pulses and one $\pi$ pulse. Further, the theory of $S U(2)$ turns has helped to clarify the deep relationship between the structure of this group and geometric phase in two-level systems [10]. It should be of interest to carry out similar geometric studies for the Lorentz group using the theory developed here.

In this work we have restricted ourselves to simply demonstrating that a consistent theory of turns is possible for the Lorentz group. The applications considered served the limited purpose of showing the effectiveness of the theory. More elaboration will, of course, be needed to handle the questions raised in the last paragraph, and we plan to return to these and other issues elsewhere.

Acknowledgement: The authors wish to thank Dr. S. Arun Kumar for his assistance in preparing the illustration.

[1] W. R. Hamilton, Lectures on Quaternions (Dublin, 1853).

[2] L. C. Biedenharn and J. D. Louck, Angular momentum in quantum physics, in Encyclopedia of Mathematics and its Applications (Addison-Wesley, Reading, MA, 1981).

[3] R. Simon, N. Mukunda, and E. C. G. Sudarshan, Phys. Rev. Lett. 62, 1331(1989).

[4] R. Simon, N. Mukunda, and E. C. G. Sudarshan, J. Math. Phys. 30, 1000 (1989).

[5] R. Simon, N. Mukunda, and E. C. G. Sudarshan, Pramana - J. Phys. 32, 769 (1989).

[6] R. Simon and N. Mukunda, Phys. Lett. A 138, 474 (1989).

[7] R. Simon and N. Mukunda, Phys. Lett. A 143, 165 (1990). 
[8] V. Bagini, R. Borghi, F. Gori, M. Santarsiero, F. Frezza, G. Schettini, and G. S. Spagnolo, Eur. J. Phys. 17, 279 (1996).

[9] M. V. Berry, Proc. Roy. Soc. London A 392, 45 (1984); S.Pancharatnam, Proc. Ind. Acad. Sci. A 44, 247 (1956).

[10] R. Simon, N. Mukunda, J. Phys. A: Math. Gen. 25, 6135 (1992).

[11] N. Mukunda, R. Simon and E. C. G. Sudarshan, Ind. J. Pure and Appl. Math. 19, 91 (1988).

[12] See, for example, I. M. Gelfand, R. A. Minlos, and Z. Ya. Shapiro, Representations of the rotation and the Lorentz groups and their applications (Pergamon, New York, 1963). Further references can be found in, N. Mukunda and R. Simon, J. Math. Phys. 36, 5170 (1995).

[13] L. H. Thomas, Nature 117, 514 (1926); Phil. Mag. 3, 1 (1927); E. P. Wigner, Ann. Math. 40, 149 (1939). An illuminating discussion can be found in the text book of H. Goldstein, Classical Mechanics, 2nd Edn. (Addison-Wesley, Reading, MA 1980), p.286.

[14] A. A. Ungar, Found. Phys. Lett. 1, 57 (1988), where further references can be found. R. Simon and N. Mukunda, Found. Phys. Lett. 3, 425 (1990); N. Salingaros, J. Math. Phys. 27, 157 (1986); Y.S. Kim and D. Son, J. Math. Phys. 27, 2228 (1986); W. E. Baylis and G. Jones, J. Math. Phys. 29, 57 (1988); P. K. Aravind, Am. J. Phys. 65, 634 ( 1997), and references therein. 